\title{
Indirect Phase Noise Sensing for Self-Healing Voltage Controlled Oscillators
}

\author{
S. Yaldiz ${ }^{1}$, V. Calayir ${ }^{1}$, X. Li ${ }^{1}$, L. Pileggi ${ }^{1}$, A. S. Natarajan ${ }^{2}$, M. A. Ferriss ${ }^{2}$, J. Tierno ${ }^{2}$ \\ ${ }^{1}$ Electrical \& Computer Engineering Department, Carnegie Mellon University, Pittsburgh, PA, USA, 15213 \\ ${ }^{2}$ IBM T.J. Watson Research Center, Yorktown Heights, NY, USA, 10598
}

\begin{abstract}
The push for higher performance analog/RF circuits in scaled CMOS necessitates self-healing via post-manufacturing tuning. A major challenge with self-healing systems is the efficient design of on-chip sensors that capture the performance of interest. This is particularly difficult for metrics such as phase noise that are not easily measured on-chip. We propose an indirect sensing method that exploits the correlations between the performance metrics of interest and those that can be measured using easy-to-integrate sensors. We demonstrate indirect phase noise sensing for a $25 \mathrm{GHz}$ self-healing voltage controlled oscillator (VCO) design in $32 \mathrm{~nm}$ CMOS SOI that approaches the best parametric yield achievable based on simulated results.
\end{abstract}

\section{INTRODUCTION}

Increasing process variability and decreasing voltage headroom in advanced silicon processes make analog circuit design more challenging. Traditional overdesign technique, whereby the circuit is designed to perform better than the specifications with large margins for variability, is becoming impractical for high performance circuits. Process variability and the push to higher performance cause unpredictable and unacceptable product yield that requires self-healing design methodologies based on post-manufacturing tuning [1, 2]. Self-healing design enables the circuit to calibrate itself not only for varying process but also for varying environment and circuit conditions that can degrade the performance. A major challenge in self-healing systems is the efficient design of onchip sensors that capture the performance of interest. Phase noise variability in voltage controlled oscillators (VCO) is an extreme example of such a challenge.

The phase noise of a VCO quantifies the spectral purity of the oscillator's output and can be a demanding specification, particularly for high data-rate wireless transceivers. For example, statistical transistor-level simulation of a $25 \mathrm{GHz}$ differential Colpitts VCO implemented in $32 \mathrm{~nm}$ CMOS SOI technology shows more than $10 \mathrm{~dB}$ variation in phase noise across temperature (-20 to $80 \mathrm{C})$, frequency $(19.5$ to $28.5 \mathrm{GHz})$ and process variations (see Fig. 1). For the VCO considered here, the bias voltage, which controls the bias current of the VCO, can be tuned externally and 3-bit digitally switched capacitor banks enable coarse frequency tuning. Fig. 1 shows the phase noise at $10 \mathrm{MHz}$ offset across bias voltage for several process, temperature and frequency settings. Simulations indicate that there is no optimum bias voltage that can guarantee minimum phase noise under all process and temperature corners. Clearly, there is a need for self-healing to mitigate variations in VCO phase noise.

Automatic amplitude control (AAC) techniques have been previously presented to reduce variations in oscillation amplitude [3]. However, AAC does not completely solve the phase noise variability problem, as the phase noise does not correlate perfectly with amplitude. Control loop stability and the variations in the amplitude detectors also limit the efficacy of AAC.

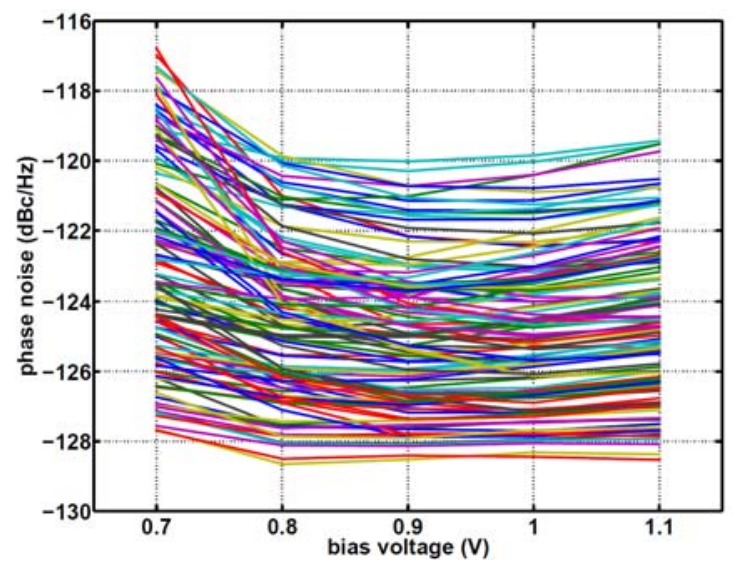

Fig. 1. Variability in VCO phase noise due to bias voltage, process, environment and frequency setting.

Integrated phase-noise sensors for phase-noise minimization and testing after manufacturing have also been presented in the literature [4]. However, these sensors cannot be used for applications where the noise specification is close or below the noise floor of the integrated sensor. For example, the phase noise sensor in [4], achieves $-75 \mathrm{dBc}$ sensitivity at $100 \mathrm{kHz}$ offset from a $2 \mathrm{GHz}$ carrier, corresponding to a sensitivity of $-95 \mathrm{dBc}$ at $10 \mathrm{MHz}$ offset from a $20 \mathrm{GHz}$ carrier.

We propose indirect phase noise sensing to enable selfhealing VCO design. Indirect sensors exploit the correlations between the VCO phase noise and circuit parameters or other performance metrics that are already available or can be measured using easy-to-integrate sensors. The indirect sensor is based on response surface modeling that has been successfully applied to model performance metrics over multidimensional variation space [5]. Response surface modeling enables us to collect and use correlation data in a compact form. The proposed indirect sensor can accurately predict the phase noise over process and temperature variations for different bias and frequency settings, and can be evaluated easily by an on-chip computing unit. We present the design methodology for indirect phase noise sensor in Section II.A. Furthermore, we present an optimization formulation that reduces the on-chip integration cost and the evaluation time of the proposed indirect sensor in Section II.B. 
Based on the indirect noise sensing, we propose a selfhealing VCO design (shown in Fig. 2) that provides the proper bias to the VCO, autonomously mitigating the effects of process and temperature variability on phase noise. The selfhealing design can either find the lowest VCO bias that achieves targeted phase noise or operate the $\mathrm{VCO}$ at minimum phase noise. In Section III, we demonstrate the efficacy of self-healing VCO design using statistical circuit simulations. We compare the self-healing VCO design with the traditional AAC technique and the optimum algorithm that employs an ideal phase noise sensor. Section IV presents our conclusions.

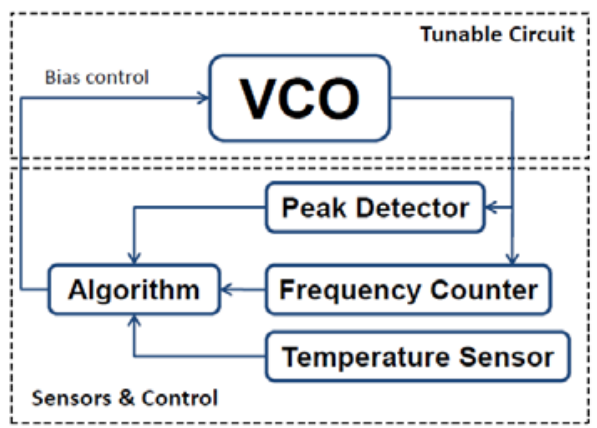

Fig. 2. Self-healing VCO design with control algorithm based on indirect sensing.

\section{INDIRECT PHASE NoISE SENSING}

\section{A. Design Methodology}

The design of indirect phase-noise sensing begins with simulation data collection over the joint space of process, temperature, frequency setting and bias voltage. Data collection in process space is achieved by running transistorlevel Monte Carlo simulations. Data collection in temperature, frequency setting and bias voltage space is achieved by variable sweeps available in circuit simulators. For each simulated sample in this joint space, phase noise, free-running oscillation frequency and amplitude are recorded. The data set generation does not introduce a significant cost since such simulations are typically required for pre-silicon performance verification.

Both the simulated frequency and amplitude values in the data set are quantized to capture the quantization effects of actual sensors. The amplitude is quantized with 5-bit resolution that yields a bin size of $80 \mathrm{mV}$. The frequency is quantized with a bin size of $300 \mathrm{MHz}$. The oscillation frequency can easily be measured using a frequency counter. The oscillation amplitude and temperature can be measured by previously presented on-chip sensors ([3], [9]).

Although analytical expressions for phase noise have been presented in the literature, such expressions are either based on simplifying assumptions or involve parameters that cannot be measured [6]. Therefore, we employ nonlinear regression to predict phase noise where the phase noise is approximated as a polynomial function as follows:

PhaseNoise $=f\left(k_{B A N D}\right.$, BiasVoltage, Amplitude, Temperature, Frequency $)(1)$ where $k_{B A N D}$ represents the value of 3-bit binary code controlling the frequency band. The polynomial coefficients in (1) are obtained by solving the unconstrained convex problem that minimizes the root mean squared (rms) error between the predicted and simulated phase noise over $\mathrm{N}$ samples as follows:

$$
\underset{\alpha}{\operatorname{minimize}}\|\mathrm{M} \alpha-\mathrm{P}\|_{2}^{2}
$$

$\mathrm{M}$ is an N-by-c matrix where each row contains the values of polynomial terms for each sample, $\alpha$ is a c-by-1 vector of unknown coefficients and $\mathrm{P}$ is an N-by-1 vector of simulated phase noise for each sample. The polynomial order, and thereby the number of coefficients denoted by c, depends on the phase noise sensitivity of the VCO design to observed performance metrics and is minimized to reduce implementation complexity.

The accuracy of indirect sensing is evaluated by 10 -fold cross validation ([7]) using 10k samples. At each iteration of the cross validation, the data set is partitioned into training and validation subsets. The training set is used for nonlinear regression while the validation set that is not used during regression is used to evaluate prediction accuracy. Fig. 3 shows that a second order polynomial model with logarithm transform provides sufficient accuracy for the $25 \mathrm{GHz} \mathrm{VCO}$ design considered in this work. Fig. 4 compares the phase noise predicted by indirect sensor with the simulated phase noise for a randomly generated validation set. The indirect sensor can predict the phase noise with an rms error of $0.5 \mathrm{dBc} / \mathrm{Hz}$ and with a maximum error of $2.0 \mathrm{dBc} / \mathrm{Hz}$ where the total phase noise variation in the data set is up to $15 \mathrm{dBc} / \mathrm{Hz}$. The quantization in measured performance metrics, namely the amplitude, frequency and temperature, has a negligible impact on the prediction accuracy.

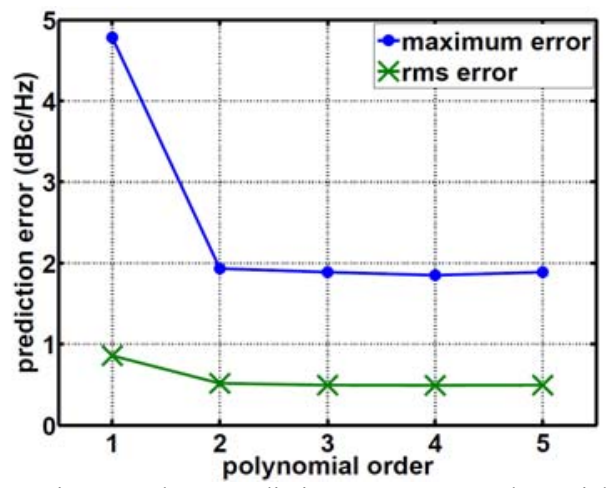

Fig. 3. Maximum and rms prediction error versus polynomial order.

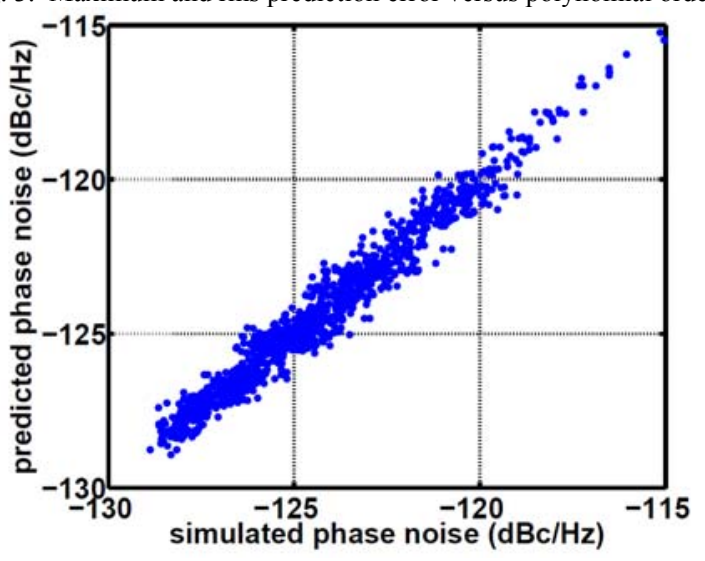

Fig. 4. Predicted versus simulated phase noise. 


\section{B. Optimization of Indirect Performance Sensors}

Polynomial regression that is obtained by solving the optimization problem stated in (2) yields a polynomial model with a non-zero coefficient for each polynomial term. For example, the solution of (2) for a quadratic model of 5 input variables result in 21 non-zero coefficients to be stored onchip. However, it is possible that some of these polynomial terms may be redundant with negligible contribution to prediction accuracy. If one can determine and eliminate such insignificant polynomial terms, both the on-chip memory overhead and the computation overhead of the indirect sensing can be reduced. To optimize an indirect performance sensor for on-chip integration, we re-formulate the problem in (2) as an $\mathrm{L}_{1}$-norm minimization problem as follows:

$$
\begin{array}{ll}
\underset{\alpha, \beta}{\operatorname{minimize}} & \|M \alpha-P\|_{2}^{2} \\
\text { subject to } & \beta_{1}+\beta_{2}+\ldots+\beta_{N} \leq \lambda \\
& \alpha_{i} \leq\left|\beta_{i}\right| \quad(i=1,2, \ldots, c)
\end{array}
$$

where $\lambda$ is the regularization factor that determines the sparsity of the solution [8]. The regularization factor in (3) is determined by cross validation and enables trade off of prediction accuracy for integration overhead by yielding fewer polynomial terms.

TABLE I

Comparison of full and optimized performance sensors

\begin{tabular}{|l|c|c|c|}
\hline & $\begin{array}{c}\text { \# of model } \\
\text { coefficients }\end{array}$ & $\begin{array}{c}\text { Maximum } \\
\text { Error }\end{array}$ & $\begin{array}{c}\text { RMS } \\
\text { Error }\end{array}$ \\
\hline Full sensor & 21 & 2.04 & 0.53 \\
\hline Optimized sensor & 13 & 2.07 & 0.55 \\
\hline Sensors optimized & 10 & 2.38 & 0.58 \\
\cline { 2 - 4 } for relaxed & 9 & 3.38 & 0.69 \\
\cline { 2 - 4 } accuracy & 8 & 3.61 & 0.73 \\
\hline
\end{tabular}

Table I compares the full indirect sensor with all model coefficients with the sensors optimized by the regularization technique for varying accuracy targets. The optimized sensor reported in the $2^{\text {nd }}$ row of Table I achieves almost the same rms error with the full sensor. This demonstrates that some of the model coefficients are indeed negligible, and therefore the integration overhead can be reduced. Table I also shows that more area-efficient sensors can be obtained at the cost of slight accuracy degradation by the formulation in (3).

\section{SElf-HeAling Algorithms AND EVAluation}

Based on the indirect phase noise sensor, we developed and evaluated two self-healing algorithms. The first algorithm utilizes the sensor to minimize the VCO bias voltage, and thereby minimizes power dissipation, while achieving a given phase noise target. We refer to this algorithm as powerminimized self-healing. The second algorithm adjusts VCO bias voltage to achieve minimum phase noise. We refer to this algorithm as noise-minimized self-healing. For our particular VCO design, we employed an incremental search for both algorithms since phase noise is a quasi-convex function of the bias voltage (due to the amplitude saturation in LC oscillators [6]) and power dissipation increases monotonically with the bias voltage. This quasi-convexity guarantees that an incremental search algorithm can find the global optimum.

To evaluate the algorithms, we simulated the VCO for three different temperatures from -20 to $80 \mathrm{C} ; 3$ different frequency settings; and 5 different bias voltages from 0.7 to $1.1 \mathrm{~V}$. For each combination of these parameters, the VCO is simulated for 300 Monte Carlo samples yielding a total of $13.5 \mathrm{k}$ samples. In the following comparisons, the power dissipation reflects the average power dissipation across process, temperature and frequency settings. The parametric yield refers to the probability that the algorithm can achieve the phase noise target for any temperature and frequency setting. We compared the power-minimized self-healing VCO design that utilizes the indirect phase noise sensing against the following approaches:

- Optimum is an unrealizable algorithm that employs an ideal phase noise sensor and, therefore can determine the best achievable phase noise and the minimum bias voltage to achieve target phase-noise. The optimum represents the upper bound on parametric yield and the lower bound on the average power dissipation for a given phase noise target.

- Fixed bias represents a design without any tuning capabilities and utilizes a fixed VCO bias for varying phase-noise targets. For a fair comparison, a unique bias voltage is selected for each phase noise target to achieve the best parametric yield and minimum power dissipation.

- AAC is the traditional automatic amplitude control algorithm that maintains the oscillation amplitude at a predefined level [3]. For a fair comparison, a unique amplitude target is selected for each phase noise target to achieve the best parametric yield and minimum power dissipation.

Fig. 5 compares the simulated parametric yield of the algorithms listed above for varying phase noise target. Fig. 5 shows that fixed bias, AAC and the power-minimized selfhealing are very close to the optimum performance. As the performance specification becomes more aggressive (below $120 \mathrm{dBc} / \mathrm{Hz}$ ), the simulated parametric yield declines sharply for all algorithms since the phase noise specification can no longer be achieved for all temperature and frequency setting.

Fig. 6 compares the average power dissipation of the optimum, fixed bias and AAC normalized to the powerminimized self-healing design. Although Fig. 5 shows that the fixed bias approach achieves parametric yield similar to selfhealing, it results in 25 to 60 percent more power dissipation compared to self-healing. The self-healing design offers a significant reduction in power dissipation compared to fixed bias circuit by applying only as much bias voltage as is necessary given the process corner, environment temperature and frequency setting. It is also noteworthy that the power dissipation achieved by self-healing is within $10 \%$ of the optimum. The difference in power dissipation by optimum and self-healing is due to the error margin added to the predicted noise to achieve high parametric yield. 
Fig. 7 compares the phase noise achieved by the noiseminimized self-healing design with the minimum phase noise achieved by the optimum. Fig. 7 shows that noise-minimized self-healing yields performance that approaches the optimum operating point (within $0.04 \mathrm{dBc} / \mathrm{Hz}$ on the average and within $0.7 \mathrm{dBc} / \mathrm{Hz}$ in the worst-case), demonstrating the accuracy of indirect sensing.

\section{CONCLUSION}

In this paper we proposed indirect phase noise sensing to predict the phase noise using readily available circuit parameters and other easy-to-measure performance metrics in the presence of process and environment variations. We presented a design methodology for indirect sensors that also minimizes on-chip integration cost. Utilizing indirect sensing, we demonstrated two self-healing algorithms for phase noise or power minimization and evaluated their efficacy using simulations. Self-healing based on indirect sensing offers a viable solution for high performance design in advanced silicon processes.

\section{ACKNOWLEDGMENTS}

This work is sponsored by the DARPA HEALICS (SelfHealing Mixed-Signal Integrated Circuits) program under Air Force Research Laboratory (AFRL) contract FA8650-09-C7924. The views expressed are those of the author and do not reflect the official policy or position of the Department of Defense or the U.S. Government.

\section{REFERENCES}

[1] Keskin, G.; Proesel, J.; Pileggi, L., "Statistical modeling and post manufacturing configuration for scaled analog CMOS," Custom Integrated Circuits Conference (CICC), 2010 IEEE, pp.1-4, Sept. 2010

[2] Natarajan, V.; Sen, S.; Banerjee, A.; Chatterjee, A.; Srinivasan, G.; Taenzler, F.; , "Analog Signature- Driven Postmanufacture Multidimensional Tuning of RF Systems," Design \& Test of Computers, IEEE , vol.27, no.6, pp.6-17, Nov.-Dec. 2010

[3] Maxim, A., "A multi-rate 9.953-12.5-GHz 0.2- $\mu \mathrm{m}$ SiGe BiCMOS LC oscillator using a resistor-tuned varactor and a supply pushing cancellation circuit," Solid-State Circuits, IEEE Journal of, vol.41, no.4, pp.918-934, April 2006

[4] Khalil, W.; Bakkaloglu, B.; Kiaei, S., "A Self-Calibrated On-Chip Phase-Noise Measurement Circuit With -75 dBc Single-Tone Sensitivity at $100 \mathrm{kHz}$ Offset," Solid-State Circuits, IEEE Journal of, vol.42, no.12, pp.2758-2765, Dec. 2007

[5] Wang, J.; Yaldiz, S.; Li, X.; Pileggi, L., "SRAM parametric failure analysis," Design Automation Conference, 2009. DAC '09. 46th ACM/IEEE, pp.496-501, 26-31 July 2009

[6] Lee, T. H., "The Design of CMOS Radio-Frequency Integrated Circuits", Cambridge University Press, December 2003

[7] Hastie, T.; Tibshirani, R.; Friedman, J., The Elements of Statistical Learning, Springer, 2003

[8] Li, X., "Finding Deterministic Solution From Underdetermined Equation: Large-Scale Performance Variability Modeling of Analog/RF Circuits," Computer-Aided Design of Integrated Circuits and Systems, IEEE Transactions on, vol.29, no.11, pp.1661-1668, Nov. 2010

[9] Woo, K.; Meninger, S.; Xanthopoulos, T.; Crain, E.; Dongwan Ha; Ham, D.; , "Dual-DLL-based CMOS all-digital temperature sensor for microprocessor thermal monitoring," Solid-State Circuits Conference Digest of Technical Papers, 2009. ISSCC 2009. IEEE International, vol., no., pp.68-69,69a, 8-12 Feb. 2009

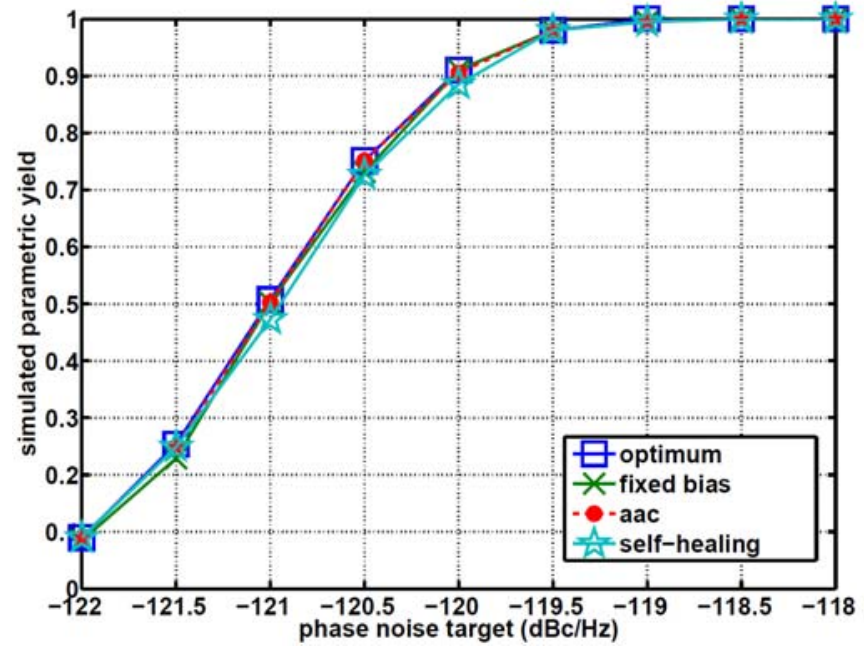

Fig. 5. Simulated parametric yield of phase noise achieved by optimum, fixed bias, AAC and power-minimized self-healing.



Fig. 6. Simulated power dissipation of optimum, fixed bias and AAC

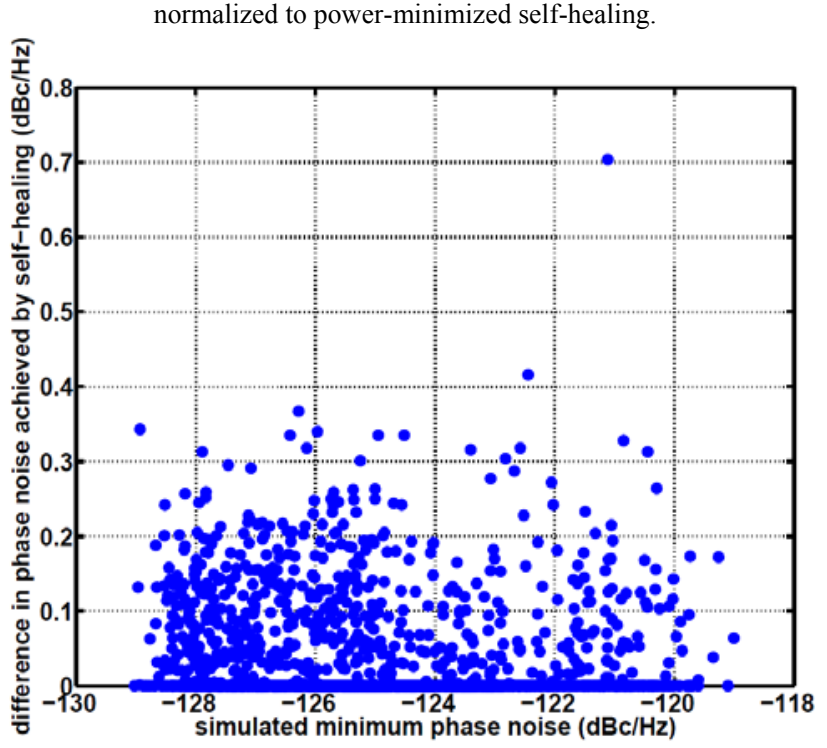

Fig. 7. The difference between the phase noise achieved by noise-minimized self-healing and minimum phase noise. 